Departamento de Neurología. Escuela de Medicina. Pontificia Universidad Católica de Chile. Santiago, Chile.

Los autores declararon no tener conflictos de intereses, utilizando el ICMJE Uniform Format for Declaration of Potential Conflicts of Interest.

Recibido el 20 de diciembre de 2012, aceptado el 30 de octubre de 2013

Correspondencia a:

Dr. Felipe Suárez H. Marcoleta 352, Santiago, Chile.

Teléfono: 3543316. E-mail: felisuarezh@gmail. com

\section{Trombosis de seno venoso secundaria a traumatismo encéfalo craneano: caso clínico}

\author{
FELIPE SUÁREZ H., PATRICIO MELLADO T., JAIME GODOY F.
}

\section{Cerebral vein thrombosis secondary to closed head injury. Report of one case}

Intracranial sinus thrombosis (IST) after closed head injury is an uncommon but potentially serious complication. It has no correlation with the severity of the injury. The symptoms and clinical course are highly variable. The most frequent but least specific symptom is severe headache. Cerebral lesions and neurologic signs develop in half of patients with IST. We report a 29 year-old male who had an IST after a severe closed head injury. The patient initially developed headache and had later 2 secondarily generalized seizures. The magnetic resonance imaging showed a superior sagittal sinus thrombosis. Anticoagulation with unfractionated heparin and intravenous phenytoin was started. At the moment of this report he is asymptomatic and continues with oral anticoagulants and phenytoin.

(Rev Med Chile 2013; 141: 1598-1601)

Key words: Anticoagulants; Head injuries; Sinus thrombosis, intracranial.
L a trombosis de seno venoso es una causa infrecuente de ataque cerebrovascular, que representa aproximadamente 0,5 a $1 \%$ del total, y que afecta principalmente a individuos jóvenes ${ }^{1-4}$. Los factores de riesgo se dividen en: adquiridos (cirugía, trauma, embarazo, puerperio, síndrome antifosfolípidos, cáncer, hormonas exógeneas) o congénitos (trombofilias) ${ }^{1,2}$. La trombosis de seno venoso secundaria a traumatismo encéfalo craneano es reportada a nivel mundial como casos únicos o series pequeñas ${ }^{3,4}$.

La cefalea es el síntoma más frecuente (90\%), con intensidad progresiva en días a semanas y escasa respuesta a analgésicos. Una minoría debuta como cefalea ictal. Las focalizaciones neurológicas más frecuentes son afasia y hemiparesia, revelando una complicación local, ya sea infarto venoso o hemorragia. Papiledema se presenta en $25 \%$ de los casos y crisis epilépticas en cerca de $40 \%^{1,4}$. El cuadro se confirma mediante T1 en corte fino con gadolinio por resonancia magnética (RM) o con tomografía computarizada (TC) $)^{1,2}$.

\section{Caso clínico}

Paciente de 29 años, sin antecedentes relevantes. Diez días antes del ingreso sufrió agresión en vía pública con objeto contundente, golpes de puño y patadas en el cráneo, sin compromiso de conciencia. Fue llevado al Servicio de Urgencia de nuestro hospital, donde se constató un examen neurológico normal y se realizó una TC de encéfalo sin contraste que resultó normal. Fue dado de alta a domicilio con antiinflamatorios no esteroidales y evolucionó con cefalea bifrontal intermitente asociada a náuseas. El día del ingreso presentó giro cefálico y desviación conjugada de mirada a izquierda, acompañado por ruido gutural, seguida de elevación tónica y simétrica de extremidades superiores de un minuto de duración y, confusión por diez minutos. Ingresó al Servicio de Urgencia de nuestro hospital en buenas condiciones generales, afebril y hemodinámicamente estable. En el examen general destacaba una fractura de incisivo que había sucedido diez días antes. Al examen 

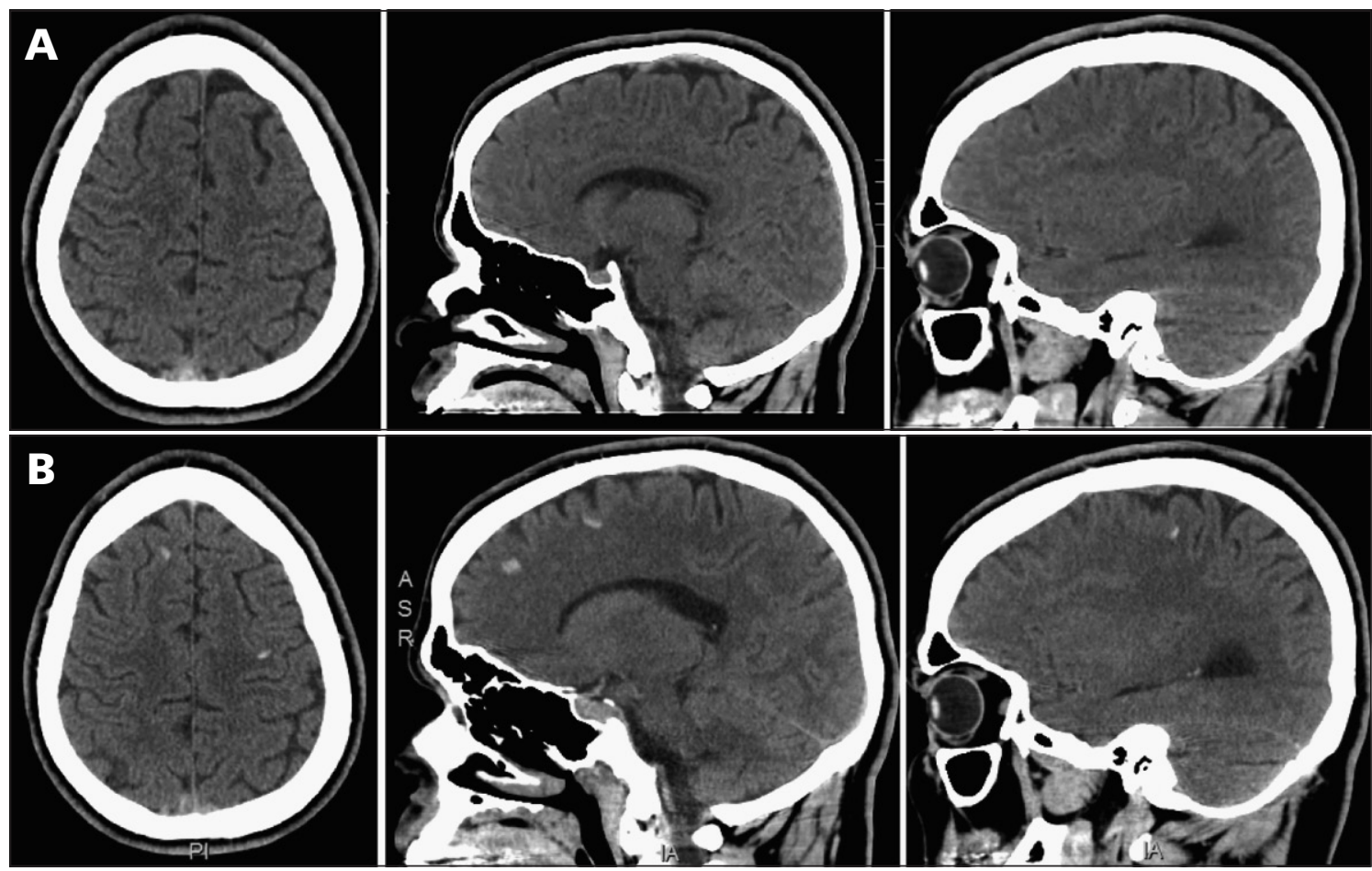

Figura 1. A. TC de encéfalo sin contraste normal $4 \mathrm{~h}$ después del TEC. B. TC de encéfalo 10 días después del TEC que muestra lesiones hemorrágicas córtico-subcorticales frontales bilaterales.
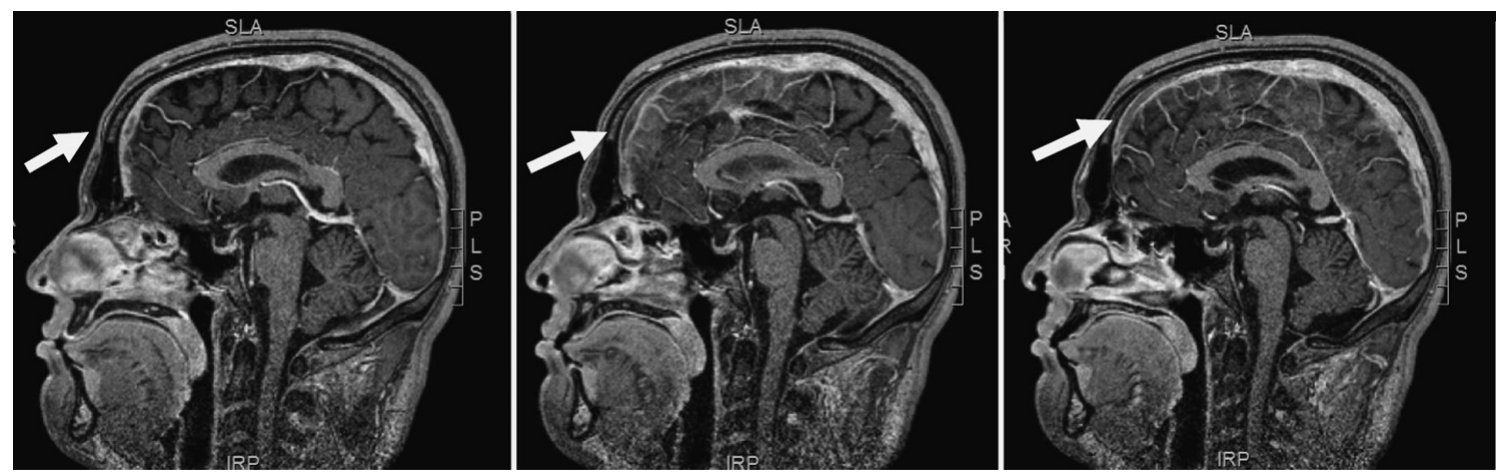

Figura 2. RM de encéfalo con gadolinio, corte fino sagital de la fase venosa: las flechas indican una trombosis de la parte anterior del seno longitudinal superior.

neurológico: bradipsíquico, sin otra alteración. El equipo de urgencia solicitó nueva TC de encéfalo sin contraste, que reveló 3 hemorragias corticosubcorticales frontales derecha y una izquierda, sin signos de fractura (Figura 1). Un EEG resultó normal. Se completó estudio con RM de encéfalo con gadolinio y $\mathrm{T} 1$ sagital volumétrico por sospecha de trombosis venosa subyacente, que confirmó la presencia de hemorragias cortico-subcorticales en los sitios anteriormente descritos, además de una trombosis del seno longitudinal superior, en su porción frontal (Figura 2). Al término de la RM presentó una nueva crisis semejante a la descrita, motivo por el cual se decidió iniciar fenitoína intravenosa $(20 \mathrm{mg} / \mathrm{kg})$ y se inició anticoagulación con heparina en bomba de infusión. Evolucionó 
satisfactoriamente, sin nuevas crisis, con traslape a anticoagulación oral con acenocumarol. Fue dado de alta a los ocho días, asintomático, condición que se mantuvo hasta su último control, seis semanas después del evento traumático. Evolucionó estable, sin cefalea ni déficit neurológico residual, desarrollando su vida normalmente.

\section{Discusión}

El caso presentado tuvo características clínicas típicas de TSV: cefalea pertinaz y crisis epilépticas focales, tuvo confirmación diagnóstica con RM y recibió terapia anticoagulante, la que se estimó no representaba un riesgo significativo para el paciente. Su evolución benigna también correspondió a un curso frecuente en esta condición.

La TSV posterior a un TEC fue descrita por primera vez en 1934 por Bagley ${ }^{3,5}$. Es una causa infrecuente de TSV que representa alrededor de $4 \%$ de todos los casos de TSV y que es más frecuente después de un TEC abierto ${ }^{6}$. No existe correlación entre la aparición de esta complicación y la intensidad del trauma ${ }^{6}$.

Aunque su fisiopatología se desconoce, diversos autores postulan como mecanismos posibles el edema citotóxico por disminución del flujo sanguíneo cerebral y el edema vasogénico, más frecuente de observar ${ }^{3}$, por hipertensión venosa retrógrada y falla consecuente de la barrera hematoencefálica. Ambos juegan un rol preponderante en el desarrollo de un infarto venoso ${ }^{6}$.

Los síntomas no son específicos, por lo cual esta entidad puede presentar un retardo en su diagnóstico o ser subdiagnosticada; tiene un amplio rango de posibles evoluciones, desde casos benignos hasta fatales ${ }^{1}$. El promedio del tiempo de inicio de una TSV post TEC en una serie de 11 pacientes fue de 4,1 días ${ }^{6}$. Algunos de los síntomas y signos característicos son cefaleas insidiosas con mala respuesta a analgésicos (90\%) o, menos frecuentes, cefalea ictal, déficit neurológicos como afasia y hemiparesia, papiledema hasta en $25 \%$ y crisis convulsivas hasta en $40 \% \%^{1,2,4}$.

La confirmación diagnóstica se obtiene mediante venografía, ya sea por resonancia o mediante TC; ambos métodos tienen resultados similares en la detección de la TSV. Si bien el T1 en corte fino con gadolinio por RM da más detalles de los senos venosos profundos y de las anomalías del parénquima ${ }^{1}$. El uso de venografía mediante TC es preferido en el caso de pacientes con marcapasos, desfibriladores, o claustrofobia y produce menos artefactos.

La recomendación actual respecto al uso de antiepilépticos, se basa en el estudio ISCVT, que sugiere tratar los pacientes con TSV que presentan alguna lesión supratentorial y que han desarrollado una convulsión ${ }^{7}$.

El manejo de la TSV post TEC es controvertido. El empleo de anticoagulantes debe ser discutido caso a caso ${ }^{5,6}$, a diferencia de la anticoagulación en TSV no traumática en la que se considera segura y efectiva, incluso en presencia de hemorragia intraparenquimatosa ${ }^{1,2,3,8}$. En los casos con curso clínico benigno, la observación clínica e imagenológica estricta, sin uso de anticoagulantes, ha tenido buenos resultados ${ }^{5}$. El tipo de anticoagulante a usar es también controvertido.

En caso de que la situación clínica empeore, el manejo médico agresivo y una eventual opción quirúrgica como la craniectomía descompresiva ante hipertensión intracraneana refractaria al tratamiento médico han sido reportadas con resultados variables ${ }^{5}$. Una revisión sistemática reciente mostró que la craniectomía descompresiva en casos seleccionados de TSV muestra mejores resultados que en el infarto maligno de la arteria cerebral media, tanto en mortalidad ( $16 \%$ versus $22 \%$ ), dependencia severa (escala de Rankin modificada $4-5,6 \%$ versus $35 \%$ ) y especialmente en recuperación completa (escala de Rankin modificada $0-1,38 \%$ versus $0 \%$ ), independiente de la edad y tiempo quirúrgico. Los pacientes en coma o con lesiones bilaterales, tuvieron resultados desfavorables, si bien, un tercio de los pacientes operados en coma logró recuperación completa ${ }^{10}$.

El tratamiento intravascular con trombectomía mecánica y balón de angioplastía ha sido usada para la TSV desde 1996, y se ha sido comunicado sólo a través de reportes de casos, con buenos resultados; se evita la trombolisis química, puesto que se reportan tasas de complicaciones hemorrágicas entre 10 a $30 \%$ de los casos ${ }^{5}$.

\section{Conclusiones}

La TSV post TEC es una causa infrecuente de TSV, pero potencialmente peligrosa si no se diagnostica oportunamente. La magnitud del trauma 
no se correlaciona con la aparición de una TSV. El síntoma más común es cefalea, seguida de crisis convulsivas o signos de focalización, los que se correlacionan habitualmente con lesiones parenquimatosas visibles en las neuroimágenes. El uso de anticoagulantes debe ser discutido caso a caso, teniendo como base que en la TSV no traumática es segura y eficaz, si bien debe tenerse en cuenta que el TEC puede aumentar las tasas de hemorragia intracraneana. En caso de deterioro neurológico debido a hipertensión intracraneana refractaria al tratamiento médico deben considerarse como alternativas las intervenciones intravasculares y la craniectomía descompresiva.

\section{Referencias}

1. Saposnik G, Barinagarrementeria F, Brown R, Bushnell C, Cucchiara B, Cushman M, et al. Diagnosis and management of cerebral venous thrombosis. Stroke 2011; 42: 1158-92.

2. Einhaupl K, Bousser MG, Brujin SFTM, Ferro J, Martinelli I, Masuhr F, et al. EFNS guideline on the treatment of cerebral venous and sinus thrombosis. Eur J Neurol 2006; 13: 553-9.
3. Bousser MG, Ferro J. Cerebral venous thrombosis: an update. Lancet Neurol 2007; 6: 162-70.

4. Stam J. Thrombosis of the cerebral veins and sinuses. N Engl J Med 2005; 352: 1791-8.

5. Bishop F, Finn M, Samuelson M, Schmidt R. Endovascular ballon angioplasty for treatment of posttraumatic venous sinus thrombosis. J Neurosurg 2009; 111: 17-21.

6. Matsushige T, Nakaoka M, Kiya K, Takeda T, Kurisu K. Cerebral sinovenous thrombosis after closed head injury. J Trauma 2009; 66: 1599-604.

7. Ferro J, Canhão P, Bousser MG, Stam J, Barinagarrementeria F. Early seizures in cerebral vein and dural sinus thrombosis. Risk factors and role of antiepileptics. Stroke 2008; 39: 1152-8.

8. Stam J, de Bruijn SFTM, deVeber G. Anticoagulation for cerebral sinus thrombosis. Stroke 2003; 34: 1054-5.

9. Van Dongen C, van den Belt A, Prins M, Lensing A. Fixed dose subcutaneous low molecular weight heparins versus adjusted dose unfractionated heparin for venous thromboembolism. Cochrane Database Syst Rev 2004; (4): CD001100.

10. Ferro J, Crassard I, Couthino J, Canhao P, Barinagarramenteria F, Cucchiara B, et al. Decompresive surgery in cerebrovenous thrombosis. A multicenter registry and a systematic review of individual patient data. Stroke 2011; 42: 2825-31. 